\title{
Will a highly developed society be able to secure population replacement?
}

\author{
Jozef Černák, ${ }^{*}$ \\ ${ }^{1}$ Department of Nuclear and Sub-Nuclear Physics, Pavol Jozef Šafárik University in Košice, \\ Slovak Republic \\ *To whom correspondence should be addressed; \\ E-mail: jozef.cernak@upjs.sk
}

In a highly developed society, economic and social changes often lead to a low total fertility rate (TFR). The low TFR has obviously negative impacts on quality of life of individuals as well as society as a whole, and it could lead to societal decline. Widening of a gap between early beginning of sexual activities and late reproduction start of individuals is a phenomenon specific to humans, not observed in apes, other mammals or other species. We believe that this gap significantly impacts the TFR, while not only economic but also social and cultural aspects like ethics, morals, and religions are important to reverse the low TFR. We contemplate social, moral and economic actions that could reduce this gap. This could also have many positive effects on personal health, satisfaction, and ensure population replacement.

Goodhart (1) warned that ignoring biological nature of fertility and population growth could cause serious issues. Divergence of the TFR decline in developing and developed counties (2) clearly shows that these warnings are justifiable. In 2005 a reversal of the TFR decline has been 
observed and linked with continuous economic and social development (2). The phenomenon gave rise to a certain optimism (3) that persists until now. However, the next study of the TFR in the period of 2010-2014 showed a transient nature of the TFR reversal (4). Assuming that the TFR is a biological phenomenon (1), and that population is a living biological system (5) with interactions between population and environment (6), we can better understand development of the TFR and propose effective actions to influence the current state.

A trend of widening of the gap between early beginning of sexual activities and late reproduction onset is a phenomenon specific to humans. It could negatively affect personal health and satisfaction, lower TFR etc.. For example, there are young people that do not want to start sexual life early, however pressure from the society is extremely high (7). On the other hand, there are adolescents which are ready to reproduce (8), obeying fundamental biological laws. However, many of them cannot realize these natural desires as a consequence of negative effects of prior life experience and environment, i.e. the society. Possible causes can be different, such as unemployment, studies, lack of resources to establish a new household, effects of sexually transmitted diseases, decreased immunity, cancers, mental disorders, difficulties to mate with a desired partner etc..

Will narrowing of this unnatural gap lead to healthier, more satisfied individuals, and societies with a safe TFR, i.e. TFR $>2.1$ (9)? Could social, moral and economic actions narrow this gap? Developed societies with under replacement levels of TFR $<2.1$ should create a new environment, i.e. economic and social norms that will stimulate healthy life, high personal satisfaction and will guarantee sustained reproduction.

The borders of the gap are defined as the age of the first sexual intercourse and the age of the first reproduction event. These individual indicators are statistically distributed, and the distributions exhibit certain shapes and mean values. Moving mean values of these distributions closer to each other will narrow the gap. This can be achieved by suitable changes of the distribution shapes.

In the developed world, beginning of sexual life, individual reproduction strategies and 
women's decisions to limit progeny number are individual choices that are to a varying extent influenced by personality and society. We propose to link these individual decisions to interactions between the human nature and the environment. From the long line of thinkers we have selected three philosophers and moralists who can be seen as representing significant ideas specific to the western culture and have a potential to bring on the expected positive changes. We note that the western society is the one most affected by the low TFR. Fragments of the chosen works help us to identify the most significant attributes of the human nature. Understanding of these attributes and their manifestations are important requirements to formulate pragmatic actions that will reduce the gap and lead to a safe TFR.

Pelagius claimed that: "Humans are responsible for their own actions and the choices they make" and "Human will is not biased towards wrong doing as a result of 'fall"" (10). JeanJacques Rousseau is frequently credited with formulating the liberal or naturalistic view of education, in its classical form postulating that human nature is not fundamentally lawless and depraved, but good, and will remain good unless it is corrupted by a corrupt society (7). Immanuel Kant conceptually defined pedagogy as consisting of discipline, civilization, cultivation and moralization. He highlighted a method of synthesis of abstract concepts and experiences into unified theories (7).

We assume that that albeit not all but many humans are naturally good, as Rousseau believed. Rousseau also believed that we have to protect young generation against the corrupt society. We believe that all humans are affected by society and education (as Rousseau and Kant stated) in both positive and negative ways. However, individuals are fully responsible for choices they make during their lives, as Pelagius claimed. This view is partially supported by studies of lethal violence that reveal an important manifestation of the human nature, showing that culture can modulate the phylogenetically inherited lethal violence in humans (11).

In accordance to Rousseau finding that timing in education is very important, we believe that strong personal responsibility in the Pelagius sense already in early age is a crucial condition to form humans that are able to resist negative influences of corrupt societies. Such 
individuals will be able to make decisions less dependent on the society. Later, individuals and society may benefit from this way of character building. However, such an approach would cost society an effort and resources, because society will have to increase quality of life of young individuals. Society will have to increase quality of formal and informal education, i.e., ensure that young individuals have an active role in the society, for example through sports, culture, and other leisure time activities. According to Rousseau it is important to guarantee free development of naturally good individuals (7), as well as appropriately influencing individuals that acquired negative features of the human nature. The ultimate goal is to strengthen individual responsibility of all young individuals.

Guaranteeing natural reproduction conditions of adolescents is rather difficult, because we need to dramatically change current economic situation of young people, such that they can make their decisions less dependent on the corrupt society (Rousseau (7)). Possible measures that can lead to changes are: decreasing mobility, reducing inequality, increasing personal savings, considering aspects of woman fertility in economic and social actions, and searching for new economic and social models that will lead to self-regulation of the TFR around the safe levels (12).

One day, in a highly developed society, the low TFR will be considered a global challenge, like those of the climate change or biodiversity loss, that require collective actions to turn the damaging behaviours into virtuous (natural) ones (13). Still, we can start today by testing the ideas of Pelagius, Rousseau and other thinkers, and, as Kant proposed, by analysing examples of morally judging reasons by repeated experiments, similar to those in chemistry, separating empirical from the rational components (7). There are huge historical records available, allowing us to test features of human nature as highlighted by Pelagius, Rousseau and Kant. We should also analyse present human activities and propose new economic, social, and education models that will take into account specific features of human nature as well as its interaction with society, i.e. the environment, to guarantee the safeTFR. 


\section{References and Notes}

1. Goodhart, C. B., Nature 178, 561 (1956).

2. M. Myrskyla, H. P. Kohler, F. C. Billari, Nature 460, 741 (2009).

3. O. Burger, J. P. DeLong, Philosophical Transactions of the Royal Society B-Biological Sciences 371 (2016).

4. J. Černák, https://arxiv.org/abs/1701.03481 (2017).

5. K. Sneppen, G. Zochi, Physics in Molecular Biology (Cambridge Univ. Press, New York, 2005).

6. G. Stulp, L. Barrett, Philosophical Transactions of the Royal Society B-Biological Sciences 371 (2016).

7. R. Curren, A Companion to the Philosophy of Education (Blackwell Publishing, Malden, 2003).

8. T. M. Smeeding, Science 346, 163 (2014).

9. D. Kirk, Population studies 50, 361(1996).

10. I. R. Fry, Dialogue between christians, jews and muslims: The concept of covenant as basis, Ph.D. thesis, MCD University of Divinity(2012). 
11. Gómez José María, Verdú Miguel, González-Megías Adela, Méndez Marcos, Nature 538, 233 (2016).

12. J. Cernak, Front. Phys. 5:11 (2017).

13. K. Nyborg, et al., Science 354, 42 (2016). 
